\title{
Association of Efimov trimers from a three-atom continuum
}

\author{
Olga Machtey, Zav Shotan, Noam Gross and Lev Khaykovich $*$ \\ Department of Physics, Bar-Ilan University, Ramat-Gan, 52900 Israel
}

\begin{abstract}
We develop an experimental technique for rf-association of Efimov trimers from a three-atom continuum. We apply it to probe the lowest accessible Efimov energy level in bosonic lithium in the region where strong deviations from the universal behavior are expected, and provide a quantitative study of this effect. The position of the Efimov resonance at the atom-dimer threshold, measured using a different experimental technique, concurs with the rf-association results.

PACS numbers: 03.75.-b, 34.50.-s, 21.45.-v
\end{abstract}

Low energy few-body physics with resonantly enhanced two-body interactions is a fundamental quantum mechanical problem, known to manifest universal properties [1]. For three interacting particles universality is associated with a ladder of weakly bound triatomic states called Efimov trimers that exist even when the twobody interactions don't support any [2]. Recent experiments with ultracold atoms have been highly successful in demonstrating several aspects of universality [3 18].

In the limit of zero collision energy, the two-body interactions are determined by a single parameter, the swave scattering length $a$, which can be tuned to very large values near a Feshbach resonance [19]. The window of universality is opened when $a$ greatly exceeds the characteristic range of the interatomic potential $r_{0}$, basically equivalent to the van der Waals length $r_{\mathrm{vdW}}=$ $\left(m C_{6} / 16 \hbar^{2}\right)^{1 / 4}$, where $C_{6}$ is the van der Waals coefficient and $m$ is the atomic mass 20]. Analytical expressions for three-body experimental observables are derived in this window in the zero range limit $\left(r_{0} \rightarrow 0\right)$ and their log-periodic dependence on $a$ reveals the discrete scaling invariance of the underlying Efimov physics [1]. The impact of the finite range of interaction potentials on the lowest Efimov energy level in the context of ultracold atoms has been considered in recent publications $21-26$ and its role in the ongoing experiments is the subject of an ongoing debate [4 6, 24 27]. Corrections to the zero range approximation can be taken into account via the effective range of the interatomic potential $R_{e}$, which is extracted from the s-wave scattering phase shift $\delta(k)$ at small relative wavenumbers $k$ using the effective range expansion $k \cot \delta(k)=-1 / a+R_{e} k^{2} / 2$. Another theoretical approach shows that deviations from universality can be directly related to the Feshbach resonance parameters 28 30].

Efimov physics in ultracold atoms is studied experimentally in the vicinity of Feshbach resonances which allow $a$ to be tuned via the external magnetic field bias [19]. The usual three-body observable which shows $\log$-periodic dependence on $a$ constitutes the three-body recombination loss rate $\left(K_{3}\right)$ of atoms from a shallow optical trap [3 16]. This experimental approach is only sensitive to a few points associated with each Efimov en- ergy level. For negative scattering length, enhancements in $K_{3}$ around certain values of $a$ enable one to probe the Efimov resonances which occur when the trimer states intersect with the three-atom continuum threshold [3]. For positive scattering length, the two lowest Efimov potentials open two pathways to a weakly bound dimer which interfere destructively for certain values of $a$, resulting in the appearance of recombination minima in $K_{3}$ spectrum [3, 5]. In addition, the signatures of the Efimov resonances at the atom-dimer threshold can be observed through secondary collision processes [5].

Here we report on a different experimental technique in which we form Efimov trimers directly from the threeatom continuum. This approach applies a strong inresonance rf-modulation of the magnetic field in order to stimulate free-atoms to trimer transition in the vicinity of the Efimov resonance at the atom-dimer threshold. At this limit the trimer is strongly asymmetric and resembles a composite dimer made up of a dimer and a nearly free atom. Fig. 1(a) shows how similar the dimer and the trimer energy levels are expected to be in this region. Near the threshold point the atom-dimer scattering length is resonantly enhanced [1] and can greatly exceed $a$. This gives the trimer wavefunction sufficient spatial extension to overlap with the free three-atom scattering state. High rates of dimer association were recently observed in our system in the relevant range of $a$ [15, 32]. Here we extend this approach and study the association of trimers. Note that the lifetime of the trimers is expected to be short, since it will either dissociate into high kinetic energy constituents (a deeply bound dimer and an atom) or an atom-trimer inelastic collision will occur. Thus, trimers disappear from the trap quickly and their binding energy shows up as a loss resonance in rffrequency scan measurements. This method provides, as a function of $a$, a continuous spectroscopy probe of the Efimov energy level and reveals its clear deviation from the predictions of universal theory. We attribute this to the manifestation of range corrections. The general trend of our data conforms with the shifted position of the Efimov resonance at the atom-dimer threshold that is detected through the secondary collision resonance in the measurements of $K_{3}$. Unfortunately, only a partial 

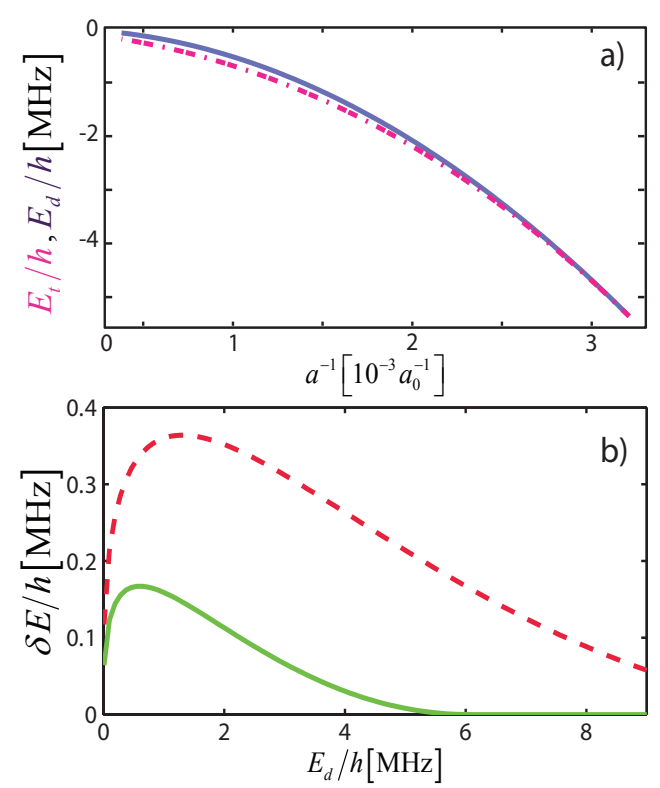

FIG. 1: (a) Dimer (solid blue) and trimer (dotted-dashed pink) energy levels follow each other very closely as calculated using the universal theory [1] with $a_{*}=288 a_{0}$, where $a_{0}$ is the Bohr radius. This value is expected from the previously measured three-body recombination spectrum [15, 32]. (b) The difference between the dimer and trimer energy levels for two different Efimov resonances at the atom-dimer threshold: $a_{*}=288 a_{0}$ (solid green) and $a_{*}=200 a_{0}$ (dashed red).

theory is currently available for bosonic lithium, and it predicts a single point in the energy spectrum - the position of the Efimov resonance at the atom-dimer threshold 25]. This prediction is in agreement with our results.

A similar experimental approach has recently been explored for fermionic ${ }^{6} \mathrm{Li}$ atoms [17, 18]. It is important to note two important differences. Primarily, extreme collisional stability of weakly bound dimers formed by two fermions [33] allows rf-association of trimers from the atom-dimer continuum [17]. This property, being the result of the Pauli exclusion principle, is not applicable in the case of bosonic ${ }^{7} \mathrm{Li}$ and makes the implementation of this experimental approach challenging. Therefore, we explore the direct association of trimers from the threeatom continuum which is more general and applicable to all particles, independent of their quantum statistical nature. Secondly, the two isotopes have very different Feshbach resonances, conveniently classified by the resonance strength parameter $s_{\text {res }}=2 r_{0} /\left|R_{e}\right|[19]$. The resonances in ${ }^{6} \mathrm{Li}$ are strongly entrance-channel dominated and thus characterized by $s_{\text {res }} \gg 1$, corresponding to hundreds of Gauss of the magnetic field range where $R_{e} \ll r_{0}$. In contrast, the resonances in ${ }^{7} \mathrm{Li}$, while being almost as broad as their ${ }^{6} \mathrm{Li}$ counterparts, are in between being entrancechannel and close-channel dominated with $s_{\text {res }} \sim 1$. This difference, caused by the anomalously large background

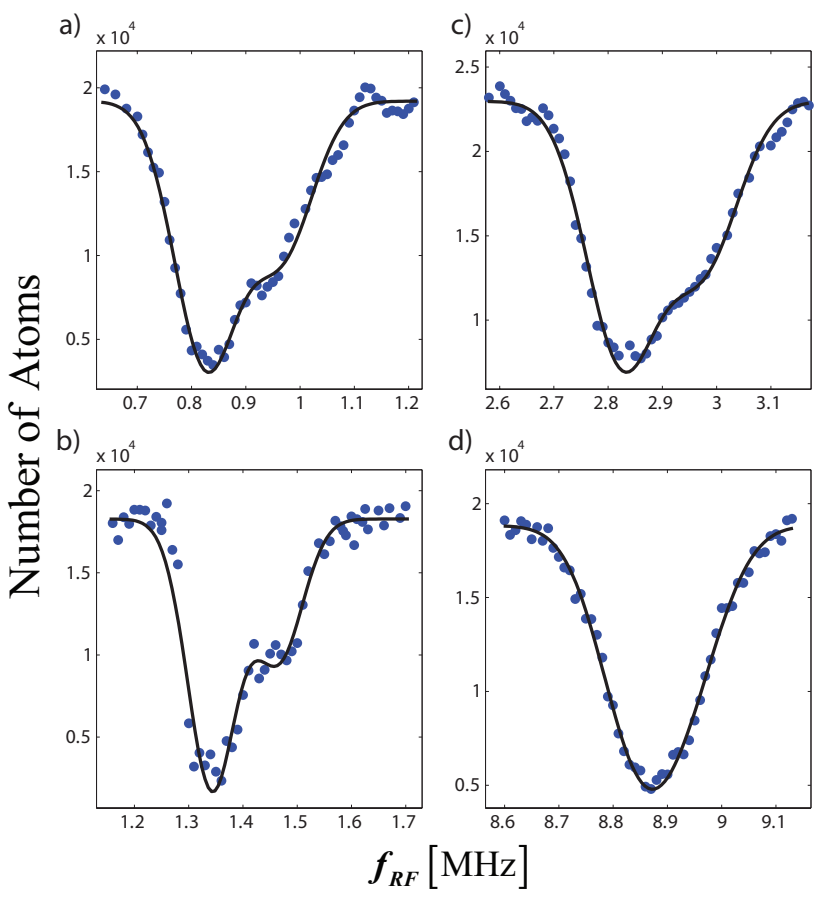

FIG. 2: Rf-induced atom loss resonances are shown for different values of the bias magnetic field corresponding to different values of the dimer binding energy. For the weakly bound dimer ((a)-(c)) strongly overlapped double-peak loss structures are clearly visible. For the deeper bound dimer (d) only a single minimum is observed, signifying that the dimer and the trimer energy levels converged to a value below the resolution limit. Each point is an average of 3 to 5 individual measurements. Solid lines represent the double-peak Gaussian fits to the data.

scattering length in ${ }^{6} \mathrm{Li}$, introduces the possibility of studying the consequences of the early breakdown of universality in two-body physics for the deviations from the universal behavior in three-body physics.

The experiments are performed on a gas of ${ }^{7} \mathrm{Li}$ atoms polarized on the $\left|F=1, m_{F}=0\right\rangle$ state and evaporatively cooled down to a typical temperature of $\sim 1.5 \mu \mathrm{K}$ in an optical trap [34]. In earlier studies of the $a$ dependent spectrum of $K_{3}$ on this state in the vicinity of a broad Feshbach resonance at $\sim 894 \mathrm{G}$ we identified a recombination minimum at $a_{0}^{*}=1130(120) a_{0}$ and a maximum at $a_{-}=-280(12) a_{0}$, where $a_{0}$ denotes the Bohr radius [7, 32]. The ratio of these two values is close to the universally predicted value of $a_{-} / a_{0}^{*}=$ $-\exp \left(-\pi / 2 s_{0}\right)=-0.21$, where $s_{0}=1.00624$, indicating that the three-body parameter is preserved across the Feshbach resonance. Using universal relations we can predict the position of the Efimov resonance at the atomdimer threshold at either $a_{*} \approx 1.1 a_{0}^{*} / \sqrt{22.7}=262(27) a_{0}$ or $a_{*} \approx-1.03 a_{-}=288(12) a_{0}$ using the recombination minimum or maximum respectively [1].

We apply a strong rf-modulation to a given magnetic field and scan its frequency to obtain atom loss reso- 
nances. Note that it corresponds to the polarization of the oscillating rf-field in the direction of the applied bias field, contrary to the orthogonal polarization required by the spin-flip transition in Refs. [17, 18]. The modulation amplitude induced by the rf-field reaches $1.6 \mathrm{G}$ at full rf-power which allows for an atom-dimer conversion efficiency of $\sim 40 \%$ within a short time of 1 msec at a typical mean density of $\sim 10^{12} \mathrm{~cm}^{-3}$ and $a \cong 1000 a_{0}$. While the rate of molecule association is defined by the two-body collision rate ( $\sim 3 \mathrm{kHz}$ for the above parameters), the trimer association rate relies on significantly rarer threebody collisions which require three atoms to be at the same point in space. In the case of a dimer, quick loss is observed when an associated dimer occasionally finds a collision partner and decays to a deeper bound state, releasing enough kinetic energy to cause the loss of all colliding parties. When the Efimov energy level is populated directly, widely separated atoms that would not necessarily collide otherwise are bound together in a weakly bound trimer state. It is then quickly dissociated into a deeply bound dimer and an atom, both of which leave the trap due to high kinetic energy. Consequently, inelastic losses are resonantly enhanced which allow us to adjust the rf-modulation time at each magnetic field value so that it is an order of magnitude less than the corresponding lifetime of the atoms due to the three-body recombination. The latter is defined as $1 / \tau=K_{3}\left\langle n^{2}\right\rangle$, where $\left\langle n^{2}\right\rangle$ is the mean of the squared atomic density [7]. The rfmodulation time varies accordingly, between $\sim 50-2000$ msec for different magnetic fields. After switching off the rf-field we detected the remaining number of atoms in the trap. A number of rf-scans for different values of the magnetic field are shown in Fig.2(a)-(d). A typical signal displays a strongly overlapping but clearly visible doublepeak loss structure in which the lower rf-frequency and deeper loss minimum correspond to the well known dimer association resonance, which we used earlier for precise characterization of the Feshbach resonance 32]. However, this signal has now been power-broadened due to the high rf-power. We associate the second loss minimum with the formation of Efimov trimers. For the low energy dimer, the trimer signal is clearly visible (Fig. 2(a)(c)). However, for the deeper bound dimer it disappears (Fig. 2(d)), as expected, when the trimer and dimer energy levels' separation are less than the typical width of the power-broadened dimer resonance $(\sim 110 \mathrm{kHz})$. Narrower dimer resonances were observed for lower rf-powers and shorter rf-modulation times but at the expense of losing the trimer signal. This trend can be observed in Fig. 2(b) which shows a narrower resonance structures accompanied by a weaker trimer signal as compared to Fig. 2(a),(c). This behavior is caused by variations in the experimental conditions of the rf-modulation pulse.

It is interesting to note that the rf-scans in Fig. 2 are well fitted with two overlapping Gaussian distributions of the same widths. Most probably this indicates that the

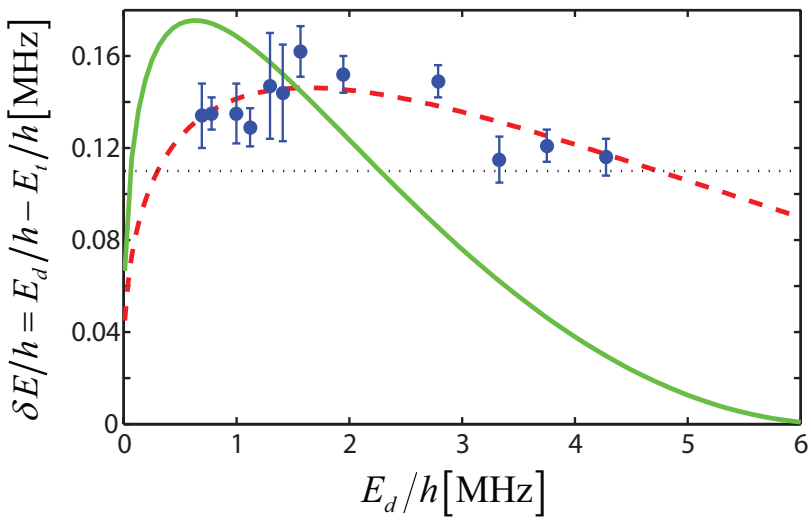

FIG. 3: The difference between trimer and dimer energies as a function of the dimer energy level. The points represent results of fits to the measurements with double-peak Gaussian distributions. The error bars show one standard deviation of the fit. The solid line represents the prediction made by universal theory for $a_{*}=288 a_{0}$. The dashed red line shows the fit of universal theory with an additional fitting parameter - the multiplication factor of the energy difference $\delta E$. The dotted horizontal line marks the minimal $\delta E$ that can be reliably detected due to the resolution limit.

trimer resonance width is not defined by the finite lifetime of trimers which should have then exceeded $10 \mu \mathrm{s}$. On the other hand, trimers do not live longer than the inverse of the two-body collision rate which limits their lifetime from above to several hundreds of $\mu$ s for the typical phase space densities used in the experiment. This range opens an experimentally realistic time slot to manipulate the trimer state. However, the direct rf-association of trimers discussed here limits their production rate to the rate of three-body collisions which is significantly slower than their decay rate that relies on two-body collisions. This might prevent the observation of macroscopic ensembles of Efimov trimers in the current approach.

We fit the rf-scans with a double-peak Gaussian distribution to determine the position of the dimer resonance, its width and the difference between the dimer and trimer energies $\delta E=E_{d}-E_{t}$. In Fig. 3, $\delta E$ is shown as a function of the dimer energy $E_{d}$. By representing only $\delta E$ we eliminate the common shift of the energy levels due to the finite temperature and strong magnetic field modulation. The points in Fig. 3 represent measurements, while the solid green line shows the prediction made by universal theory with $a_{*}=288 a_{0}$ which is derived from the $K_{3}(a)$ measurements. At first glance there appears to be a clear discrepancy between the measurements and the theory. The universal theory predicts that the trimer and the dimer energy levels will converge sooner than was observed in the experiment. For example, for $E_{d} \approx h \times 2.8 \mathrm{MHz} \delta E$ should be well below the resolution limit, preventing the observation of the trimer signal, but it is clearly visible and disappears for significantly larger $E_{d}$ (see Fig. 2(c),(d)). 
Note however, that universal theory predicts the depth of the trimer state $\delta E$ reasonably well, leaving the main disagreement between the theory and the experimental results in the convergence of the two energy levels. Our measurements then suggest that the Efimov resonance at the atom-dimer threshold is shifted to a lower scattering length value, but they also suggest that the theory needs to go beyond universality in order to correctly describe our data. If we were using a lower $a_{*}$ value within the framework of universal theory then we would obtain larger $\delta E$ in the relevant range of $E_{d}$ which clearly contradicts our results (see Fig. 1(b)). A theory of the full Efimov energy spectrum with finite range corrections for bosonic lithium does not yet exist. However, recently an effective field theory approach has been used [25] to calculate the shift of $a_{*}$ to a new position $a_{* e}$ based on $R_{e}(a)$ and $K_{3}(a)$ which were reported in our previous work [7]. This "beyond universality" theory predicts that $a_{* e}=210(44) a_{0}$ in agreement with the general trend of the experimental results. In the absence of a full theory and in order to estimate a value of $a_{* e}$ with which the measurements represented in Fig. [3 will agree, we modify the universal theory by introducing an additional parameter, a multiplication factor of the energy difference $\delta E$. We fit our data with this model and the result is shown in Fig. 3 as a dashed red line which describes our measurements reasonably well and roughly estimates $a_{* e} \approx 180 a_{0}$. Let us stress, however, that it is necessary to fit our data with a complete theory in order to correctly predict the value of $a_{* e}$.

Independently, $a_{* e}$ can be measured via the resonance from secondary collisions which is expected to appear in the $K_{3}(a)$ spectrum [5]. This resonance occurs when a dimer with relatively high kinetic energy is formed in a three-body recombination process and collides with other atoms on its way out of the trap, resulting in the loss of more than three atoms in a single three-body recombination event. Such resonance is likely to occur in the vicinity of the Efimov resonance at the atomdimer threshold where atom-dimer elastic and inelastic collisional cross-sections are enhanced by orders of magnitudes [1]. Here we reveal the $K_{3}(a)$ measurement that we performed earlier on the absolute ground state $\left(\left|F=1, m_{F}=1\right\rangle\right)$ 15, 32]. Fig. 4 shows $K_{3}(a)$ for positive scattering lengths in the vicinity of the Feshbach resonance at $\sim 738 \mathrm{G}$. In addition to the already indicated three-body recombination minimum at $a_{0}^{*}=1260(76) a_{0}$, a narrow peak appears at $\sim 200 a_{0}$. We can now attribute this peak to a secondary collision resonance that reveals the position of $a_{* e}$. To make a quantitative argument in favor of this attribution we developed a model which counts the elastic and inelastic collisions of a dimer with trapped atoms based on the available analytical expressions for the cross-sections of these events 31. Thus the model predicts enhancement of atom loss per threebody recombination event. We fit our data with this

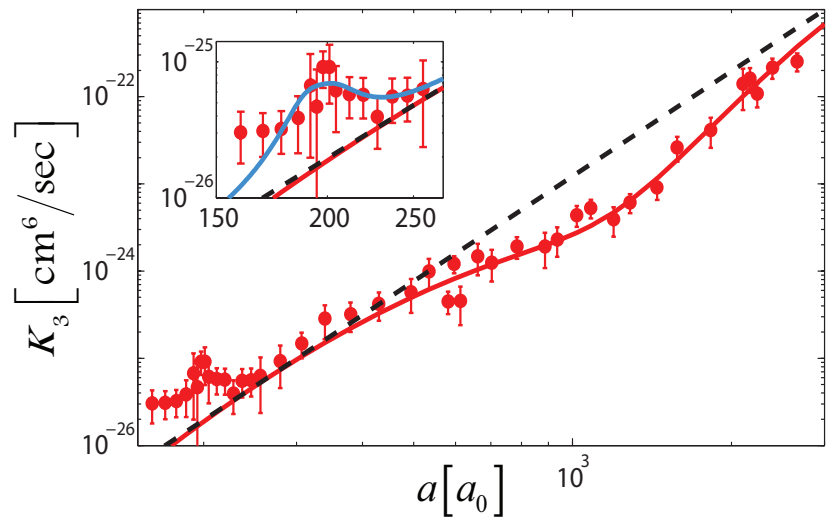

FIG. 4: The three-body recombination spectrum. Only the positive range of $a$ is shown. In addition to the earlier published minimum in $K_{3}(a)$ 15 there is a local maximum (enlarged in the inset) that we attribute to a secondary collision resonance which indicates the position of the Efimov resonance at the atom-dimer threshold $a_{e}^{*} \approx 200 a_{0}$. The solid line in the inset is a fit to the model of secondary collisions [31].

model using three fitting parameters: the height of the peak (which has no meaning as we do not model the $K_{3}$ coefficient directly), $a_{* e}$, and $\eta$ which describes the lifetime of a trimer. The fit, shown as a solid line in the

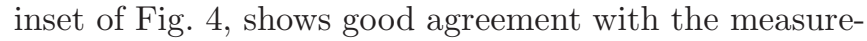
ment. This supports our treatment of the feature as an avalanche resonance. We note that the fitting parameter $\eta=0.072(1)$ is smaller by a factor of $\sim 2.5$ compared to the experimentally observed values for the minimum and maximum in the $K_{3}(a)$ spectrum[15, 32]. This disagreement might be attributed to yet another manifestation of the "beyond universality" corrections required to explain our experimental results.

The fitted position of $a_{* e}=196(4) a_{0}$ does not agree with the universal theory's prediction of either $a_{*}=$ $292(18) a_{0}$ or $a_{*}=282(12) a_{0}$ using $a_{0}^{*}$ or $a_{-}$, respectively. However, it does agree with the general trend of the rf-association results performed on a different state, which predict the shift of $a_{*}$ to lower values as well. Remarkably, the measured position of $a_{* e}$ in the absolute ground state corresponds well to the value predicted by the "beyond universality" theory for the second to lowest Zeeman sublevel 25]. This similarity may not be surprising since the other Efimov features were discovered to be identical for both levels within the experimental errors [15, 32]. Moreover, it was shown that the effective range $R_{e}$ and the parameters of the Feshbach resonances on both levels differ insignificantly [7, 25, 32]. However, as we noted earlier, the fitting of our rf-association measurements to a complete theory will allow a quantitative comparison between the values of $a_{* e}$ and, thus, a detailed analysis of the influence of the finite range corrections on both levels. We note, finally, that our original $K_{3}(a)$ measurements on the $\left|F=1, m_{F}=0\right\rangle$ level [7] 
were not dense enough in the relevant region of $a$ to allow the identification of a similar avalanche resonance and they have to be revisited to reveal this feature.

It is interesting to compare our results with those obtained for ${ }^{6} \mathrm{Li}$, especially in light of the experimentally observed universality in the position of the Efimov resonance at the free atom threshold $\left(a_{-}\right)$in the region of $a<0$. In all but one atomic species $a_{-} / r_{\mathrm{vdW}} \sim-9.5$ within $\pm 15 \%$ [5 $7,10-13,15,16,35]$. This was recently explained in a landmark theoretical paper 36, 37]. Despite this universality in $a_{-}, a_{* e}$ varies significantly between different species [4, 5, 13, 14, 31] which is a clear consequence of corrections caused by the finite range of interatomic potentials. However, though $r_{\mathrm{vdW}}$ for both isotopes of Li is very similar [19], $a_{* e}$ still differs significantly. In our case $a_{*} / a_{* e} \sim 1.5$, but for ${ }^{6} \mathrm{Li}$ corrections go in the opposite direction, i.e. $a_{*} / a_{* e}<1$ [13, 14]. This emphasizes the role played by the $s_{\text {res }}$ parameter in the breakdown of three-body universality in ${ }^{7} \mathrm{Li}$.

In conclusion, we developed a general experimental technique which paves the way for direct population, manipulation and interrogation of Efimov quantum states. During the short but experimentally reasonable lifetime of Efimov trimers, they can be moved into a deeply bound state and their macroscopic ensemble can then be realized. We also note that a recent theory has found reasonable rf-association rates of Efimov trimers in ${ }^{7} \mathrm{Li}$ for similar phase space densities [38]. However, it uses a different starting configuration (one boson is distinguishable) and thus cannot be directly applied to our system.

LK acknowledge stimulating discussions with L. Platter, E.A. Cornell, E. Braaten and S. Jochim. We thank S.J.J.M.F. Kokkelmans for many inspiring discussions and critical reading of the manuscript. This work was supported, in part, by the Israel Science Foundation.

* Electronic address: lev.khaykovich@biu.ac.il

[1] E. Braaten and H.-W. Hammer, Phys. Rep. 428, 259 (2006).

[2] V. Efimov, Phys. Lett. B 33, 563 (1970).

[3] T. Kraemer, M. Mark, P. Waldburger, J. G. Danzl, C. Chin, B. Engeser, A. D. Lange, K. Pilch, A. Jaakkola, H.-C. Nägerl, R. Grimm, Nature 440, 315 (2006).

[4] S. Knoop, F. Ferlaino, M. Berninger, M. Mark, H.-C. Nägerl, and R. Grimm, Nature Phys. 5, 227 (2009).

[5] M. Zaccanti, B. Deissler, C. D'Errico, M. Fattori, M. Jona-Lasinio, S. Müller, G. Roati, M. Inguscio, and G. Modugno, Nature Phys. 5, 586 (2009).

[6] S. E. Pollack, D. Dries, and R. G. Hulet, Science 326, 1683 (2009).

[7] N. Gross, Z. Shotan, S. Kokkelmans, and L. Khaykovich, Phys. Rev. Lett. 103, 163202 (2009).

[8] G. Barontini, C. Weber, F. Rabatti, J. Catani, G. Thalhammer, M. Inguscio, and F. Minardi, Phys. Rev. Lett. 103, 043201 (2009).
[9] F. Ferlaino, S. Knoop, M. Berninger, W. Harm, J. P. D'Incao, H.-C. Nägerl, and R. Grimm, Phys. Rev. Lett. 102, 140401 (2009).

[10] T. B. Ottenstein, T. Lompe, M. Kohnen, A. N. Wenz, and S. Jochim, Phys. Rev. Lett. 101, 203202 (2008).

[11] J. H. Huckans, J. R. Williams, E. L. Hazlett, R. W. Stites, and K. M. O'Hara, Phys. Rev. Lett. 102, 165302 (2009).

[12] J. R. Williams, E. L. Hazlett, J. H. Huckans, R. W. Stites, Y. Zhang, and K. M. O'Hara, Phys. Rev. Lett. 103, 130404 (2009).

[13] T. Lompe, T. B. Ottenstein, F. Serwane, K. Viering, A. N. Wenz, G. Zürn, and S. Jochim, Phys. Rev. Lett. 105, 103201 (2010).

[14] S. Nakajima, M. Horikoshi, T. Mukaiyama, P. Naidon, and M. Ueda, Phys. Rev. Lett. 105, 023201 (2010).

[15] N. Gross, Z. Shotan, S. Kokkelmans, and L. Khaykovich, Phys. Rev. Lett. 105, 103203 (2010).

[16] M. Berninger, A. Zenesini, B. Huang, W. Harm, H.-C. Nägerl, F. Ferlaino, R. Grimm, P. S. Julienne, and J. M. Hutson, Phys. Rev. Lett. 107, 120401 (2011).

[17] T. Lompe, T. B. Ottenstein, F. Serwane, A. N. Wenz, G. Zürn, and S. Jochim, Science 330, 940 (2010).

[18] S. Nakajima, M. Horikoshi, T. Mukaiyama, P. Naidon, and M. Ueda, Phys. Rev. Lett. 106, 143201 (2011).

[19] C. Chin, R. Grimm, P. Julienne, and E. Tiesinga, Rev. Mod. Phys. 82, 1225 (2010).

[20] G. F. Gribakin and V. V. Flambaum, Phys. Rev. A 48, 546 (1993).

[21] Earlier theoretical treatments, especially within the framework of nuclear physics, are reviewed in Ref. [1].

[22] M. Thøgersen, D. V. Fedorov, and A. S. Jensen, Phys. Rev. A 78, 020501(R) (2008).

[23] L. Platter, C. Ji, and D. R. Phillips, Phys. Rev. A 79, 022702 (2009).

[24] J. P. D'Incao, C. H. Greene, and B. D. Esry, J. Phys. B 42, 044016 (2009).

[25] C. Ji, D. Phillips, and L. Platter, Europhys. Lett. 92, 13003 (2010).

[26] P. Naidon and M. Ueda, C.R. Physique 12, 13 (2011).

[27] M. Thøgersen, D. V. Fedorov, A. S. Jensen, B. D. Esry, and Y. Wang, Phys. Rev. A 80, 013608 (2009).

[28] M. Jona-Lasinio and L. Pricoupenko, Phys. Rev. Lett. 104, 023201 (2010).

[29] L. Pricoupenko, Phys. Rev. A 82, 043633 (2010).

[30] L. Pricoupenko and M. Jona-Lasinio, Phys. Rev. A 84, 062712 (2011).

[31] O. Machtey, D. A. Kessler, and L. Khaykovich, Phys. Rev. Lett. 108, 130403 (2012).

[32] N. Gross, Z. Shotan, O. Machtey, S. Kokkelmans, and L. Khaykovich, C.R. Physique 12, 4 (2011).

[33] J. Cubizolles, T. Bourdel, S. J. J. M. F. Kokkelmans, G. V. Shlyapnikov, and C. Salomon, Phys. Rev. Lett. 91, 240401 (2003).

[34] N. Gross and L. Khaykovich, Phys. Rev. A 77, 023604 (2008).

[35] R. J. Wild, P. Makotyn, P. M. Pino, E. A. Cornell, and D. S. Jin, Phys. Rev. Lett. 108, 145305 (2012).

[36] J. Wang, J. P. D'Incao, B. D. Esry, and C. H. Greene, arXiv:1201.1176.

[37] Note that Ref. [C. Chin, arXiv:1111.1484] provides an intuitive but perhaps less strict theoretical model of universality.

[38] T. V. Tscherbul and S. T. Rittenhouse, Phys. Rev. A 84, 062706 (2011). 